\title{
GLOBAL PROPERTIES OF THE THREE-DIMENSIONAL PREDATOR-PREY LOTKA-VOLTERRA SYSTEMS
}

\author{
A.KOROBEINIKOV \\ andrei@scitec.auckland.ac.nz \\ Department of Mathematics, University of Auckland, New Zealand \\ G.C.WAKE \\ g.wake@math.canterbury.ac.nz \\ Department of Mathematics and Statistics, University of Canterbury, Private Bag 4800, Christchurch, \\ New Zealand
}

\begin{abstract}
The global properties of the classical three-dimensional Lotka-Volterra two preyone predator and one prey-two predator systems, under the assumption that competition can be neglected, are analysed with the direct Lyapunov method. It is shown that, except for a pathological case, one species is always driven to extinction, and the system behaves asymptotically as a two-dimensional predator-prey Lotka-Volterra system. The same approach can be easily extended to systems with many prey species and one predator, or many predator species and one prey, and the same conclusion holds. The situation considered is common for New Zealand wild life, where indigenous and introduced species interact with devastating consequences for the indigenous species. According to our results the New Zealand indigenous species are definitely driven to extinction, not only in consequence of unsuccessful competition, but even when competition is absent. This result leads to a better understanding of the mechanism of natural selection, and gives a new insight into pest control practice.
\end{abstract}

Keywords: Lotka-Volterra systems, Lyapunov functions.

\section{Introduction}

One of the most commonly used models of predator-prey interaction is the classical Lotka-Volterra predator-prey model

$$
\dot{x}=x(a-b y), \quad \dot{y}=y(-c+d x), \quad\left(\dot{x} \equiv \frac{d x}{d t}, \dot{y} \equiv \frac{d y}{d t}\right),
$$

where $x(t), y(t)$ are the prey and predator population biomass at time $t ; a, b, c, d$ are positive constants. Phase curves of (1) form the family of closed curves, given by the first integral

$$
U=d x-c \ln x+b y-a \ln y
$$

around the neutrally-stable steady-state $(c / d, a / b)$.

The Lotka-Volterra model indeed may be the simplest possible predator-prey model. It has been criticised as being unrealistic-mainly for its structural instability and the assumption of the unlimited growth of the prey population $x(t)$ in the 
absence of a predator. Nevertheless, it is a useful tool containing the basic properties of the real predator-prey systems, and serves as a robust basis from which it is possible to develop more sophisticated models.

The model (1) can be naturally generalised for the multi-species case. The generalisation of the Lotka-Volterra model (1) for the multi-species case retains the basic features of real ecological systems and, as will be shown, allows us to obtain valuable results that are easy to be interpreted. For the three-species predator-prey interaction two possibilities arise: the two prey-one predator system

$$
\begin{gathered}
\dot{x}_{1}=x_{1}\left(a_{1}-b_{1} y\right), \quad \dot{x}_{2}=x_{2}\left(a_{2}-b_{2} y\right), \\
\dot{y}=y\left(-c+d_{1} x_{1}+d_{2} x_{2}\right),
\end{gathered}
$$

and a two predator-one prey system

$$
\begin{gathered}
\dot{x}=x\left(a-b_{1} y_{1}-b_{2} y_{2}\right), \\
\dot{y}_{1}=y_{1}\left(-c_{1}+d_{1} x\right), \quad \dot{y}_{2}=y_{2}\left(-c_{2}+d_{2} x\right) .
\end{gathered}
$$

Here $x_{1}, x_{2}$ are prey populations, $y_{1}, y_{2}$ are predator populations, and $a_{i}, b_{i}, c_{i}, d_{i}$ are positive constants.

The equations (3) assume that the prey populations $x_{1}, x_{2}$ have not reached, and are not able to reach, their media capacity because of predation or for some other reasons, and that interspecies competition is absent or negligible. These situations (the absence of the direct competition between two prey species having a common predator) are not common but they are not unrealistic, and, we are sure, have arisen repeatedly in the past in the process of evolution. As a result of the artificial introduction of alien species there are some examples of such systems in New Zealand. The interaction of kiwi-rabbits-stoats is an example. Occupying different ecological niches (bush for the kiwi and open fields for rabbits), the rabbits and kiwi do not compete. The populations of the both species are far from reaching their media capacity as a result of predation and artificial controlling of the rabbit population. Furthermore, both the kiwi and the rabbits have the same predators: stoats, cats, minks, or any others. Hence, the kiwi-rabbit-stoat interaction can be adequately described by the equations (3).

The equations (4) describe the situation when two predator species depend on a common prey and, furthermore, these two predator species do not interact directly, they do not fight directly (not usually, at least), do not predate one the other, and do not depend one on the other as a food source. Such situations are not unusual, and can arise in many different cases.

The multi-species Lotka-Volterra predator-prey systems have been studied by many authors. In the early papers, such as [1], [2], [4], [8], [9] and others, the influence of the predation on the two competing species was studied, and showed that the presence of a predator may stabilise the ecological system which is otherwise unstable. In a number of recent papers, such as [3], [5], [6], [7], the stability and the global dynamical properties of the multi-species Lotka-Volterra systems have been studied. The most complete list of references can be found in Y.Takeuchi's book [7]. 
The main aim of this work is to demonstrate that predation may destabilise a comparatively stable system, and that species can be completely annihilated during a finite time as a result of predation only, without any competition at all. Or, in contrast, an unsuccessful predator can be driven to extinction in finite time even without any direct competition. This result leads to a new insight into the mechanism of natural selection.

LEMMA 1 The systems (3) and (4) are equivalent under inversion of time $t$.

The proof is straightforward with substitution of $\tau=-t$ in (3) or (4). The lemma makes it possible to apply the same methods to both systems (3) and (4).

We call the parameter $p_{i}=a_{i} / b_{i}$ for the prey $x_{i}$, and the parameter $q_{i}=d_{i} / c_{i}$ for the predator $y_{i}$, the capacity to survive, or the survival capacity.

In the regular case, provided by $p_{1} \neq p_{2}$ for (3) and $q_{1} \neq q_{2}$ for (4), each of the systems (3), (4) has three equilibria: the trivial one at the origin and two others on the coordinate planes $x_{i} y$ or $x y_{i}$ (the coexisting equilibria of the reduced twodimensional predator-prey systems), given by $\left(c / d_{1}, 0, p_{1}\right),\left(0, c / d_{2}, p_{2}\right)$ for $(3)$, and by $\left(q_{1}^{-1}, a / b_{1}, 0\right),\left(q_{2}^{-1}, 0, a / b_{2}\right)$ for $(4)$. There are no coexisting equilibria in the regular cases. In the pathological case, provided by $p_{1}=p_{2}$ for $(3)$, or $q_{1}=q_{2}$ for (4), both systems (3), (4) have a continuum of equilibria - the straight line given by $\left(x_{1}, \frac{c-d_{1} x_{1}}{d_{2}}, p_{1}\right)$ for $(3)$, or by $\left(q_{1}^{-1}, y_{1}, \frac{a-b_{1} y_{1}}{b_{2}}\right)$ for (4), in addition to the trivial equilibrium at the origin.

The phase space of $(3)$ is the positive octant

$$
R_{+}^{3}=\left\{\left(x_{1}, x_{2}, y\right) \in R^{3} \mid x_{1} \geq 0, x_{2} \geq 0, y \geq 0\right\}
$$

the phase space of (4) is the positive octant

$$
R_{+}^{3}=\left\{\left(x, y_{1}, y_{2}\right) \in R^{3} \mid x \geq 0, y_{1} \geq 0, y_{2} \geq 0\right\}
$$

and their coordinate planes cannot be crossed by any trajectory. Hence the twodimensional coordinate planes in $R^{3}$ are the invariant sets of the systems (3) and (4), so a solution of (3) or (4) with positive initial value remains in the positive octant $R_{+}^{3}$ forever.

\section{Main results}

THEOREM 1 The solutions of (3) with positive initial values are bounded, and, if $p_{1}>p_{2}$, then $\lim _{t \rightarrow \infty} x_{2}=0$. If $p_{1}=p_{2}$ then (3) has two independent first integrals.

Proof: For the case $p_{1} \geq p_{2}$ consider two Lyapunov functions

$$
H=\frac{d_{1} x_{1}}{b_{1}}+\frac{d_{2} x_{2}}{b_{2}}+y-\frac{c}{b_{1}} \ln x_{1}-\frac{a_{1}}{b_{1}} \ln y,
$$




$$
V=\frac{x_{2}^{b_{1}}}{x_{1}^{b_{2}}}
$$

The function $H\left(x_{1}, x_{2}, y\right)$ satisfies

$$
\dot{H}=\left(p_{2}-p_{1}\right) d_{2} x_{2} \text {. }
$$

The condition $p_{1}>p_{2}$ implies that $\dot{H}<0$ when $x_{2}>0$, and $\dot{H}=0$ when $x_{2}=0$. Hence there is no flux through the coordinate plane $x_{2}=0 ; \dot{H}<0$ implies that the phase curve of (3) initiating in the positive octant $R_{+}^{3}$ remains forever in the part of $R_{+}^{3}$ bounded by the surface $H\left(x_{1}, x_{2}, y\right)=H\left(x_{1}(0), x_{2}(0), y(0)\right)$ and the plane $x_{2}=0$, and is attracted by the coordinate plane $x_{2}=0$.

If $p_{1}=p_{2}$ then $\dot{H} \equiv 0$, and $H\left(x_{1}, x_{2}, y\right)$ is the first integral of (3).

The function $V\left(x_{1}, x_{2}, y\right)$ satisfies

$$
\dot{V}=\left(a_{2} b_{1}-a_{1} b_{2}\right) V=b_{1} b_{2}\left(p_{2}-p_{1}\right) V \equiv \Delta V,
$$

which has the solution

$$
V(t)=V(0) e^{\Delta t}
$$

So the asymptotic behaviour of $V(t)$ depends upon the sign and value of

$$
\Delta=\left(p_{2}-p_{1}\right) b_{1} b_{2} \text {. }
$$

If $p_{1}>p_{2}$, then $\Delta<0$, and $\lim _{t \rightarrow \infty} V=0$. Since the solution is bounded it implies $\lim _{t \rightarrow \infty} x_{2}=0$.

If $p_{1}=p_{2}$ then $\dot{V} \equiv 0$, and $V\left(x_{1}, x_{2}, y\right)=$ constant is the first integral of (3). Obviously, $V\left(x_{1}, x_{2}, y\right)$ and $H\left(x_{1}, x_{2}, y\right)$ are independent.

This completes the proof.

By putting $x_{2}=0$ into (5) then the equation $H=$ constant is equivalent to the first integral (2) for the two-dimensional system $\left(x_{1}(t), y(t)\right)$. The Lyapunov function of the form (5) has been used by B.-S.Goh [3], Y.Takeuchi [7] and the others.

In the case $p_{1}<p_{2}$ it suffices to change subscripts.

The following theorem for the system (4) is the analogue of Theorem 1. The proof follows from Lemma 1, or two Lyapunov functions

$$
G=x-\frac{c_{1}}{d_{1}} \ln x+\frac{b_{1}}{d_{1}} y_{1}+\frac{b_{2}}{d_{2}} y_{2}-\frac{a}{d_{1}} \ln y_{1}, \quad W=\frac{y_{2}^{d_{1}}}{y_{1}^{d_{2}}}
$$

may be considered.

THEOREM 2 The solutions of (4) with positive initial values are bounded, and, if $q_{1}>q_{2}$, then $\lim _{t \rightarrow \infty} y_{2}=0$. If $q_{1}=q_{2}$ then (4) has two independent first integrals.

In the biological context there is a critical value $\bar{x}_{i}$ for a population $x_{i}(t)$ such that the species is effectively extinct if $0<x_{i}(t)<\bar{x}_{i}$. (If $x_{i}(t)$ is the number of the 
animals then $\bar{x}_{i}=1$.) Theorems 1 and 2 imply that for the systems (3), (4) this limit can be reached in finite extinction time $t_{k}$. Equation (8) allows us to evaluate the extinction time for the species $x_{i}(t)$. Assuming $p_{1}>p_{2}$ then for the system (3) $\lim _{t \rightarrow \infty} x_{2}(t)=0$. If the initial values $x_{1}(0), x_{2}(0)$ are given, and assuming $x_{1}\left(t_{k}\right)=x_{1}(0)$, and $x_{2}\left(t_{k}\right)=\bar{x}_{2}$ then, according to $(8)$,

$$
t_{k}=\frac{1}{\Delta} \ln \frac{V\left(t_{k}\right)}{V(0)}=\frac{\ln x_{2}(0)-\ln \bar{x}_{2}}{\left(p_{1}-p_{2}\right) b_{2}} .
$$

\section{Generalisation for $n+1$-dimensional systems}

The system with $n$ prey and one predator, with the assumption that the interactions between the prey species are negligible, may be described by the equations

$$
\begin{gathered}
\dot{x}_{1}=x_{1}\left(a_{1}-b_{1} y\right), \ldots, \dot{x}_{n}=x_{n}\left(a_{n}-b_{n} y\right), \\
\dot{y}=y\left(-c+\sum_{i=1}^{n} d_{i} x_{i}\right) .
\end{gathered}
$$

The order of the subscripts for (9) is chosen so that $p_{1} \geq p_{2} \geq \ldots \geq p_{n}$, where $p_{i}=a_{i} / b_{i}$ is the survival capacity of $x_{i}$. The following extension of Theorem 1 holds for (9).

THEOREM 3 The solutions of (9) with positive initial values are bounded, and, if $p_{1}=p_{2}=\ldots=p_{m}>\max \left(p_{m+1}, \ldots, p_{n}\right)$, then (9) has $m-1$ independent first integrals, and $\lim _{t \rightarrow \infty} x_{i}=0$ for all $i \geq m+1$.

If $p_{1}>\max \left(p_{2}, \ldots, p_{n}\right)$, then $\lim _{t \rightarrow \infty} x_{i}=0$ for all $i \geq 2$.

Proof: Define a Lyapunov function

$$
H\left(x_{1}, \ldots, x_{n}, y\right)=\sum_{i=1}^{n} \frac{d_{i}}{b_{i}} x_{i}+y-\frac{c}{b_{1}} \ln x_{1}-\frac{a_{1}}{b_{1}} \ln y .
$$

The derivative of $H$ along a solution of (9) satisfies

$$
\dot{H}=\sum_{i=1}^{n}\left(\frac{a_{i}}{b_{i}}-\frac{a_{1}}{b_{1}}\right) d_{i} x_{i}=\sum_{i=1}^{n}\left(p_{i}-p_{1}\right) d_{i} x_{i} .
$$

If $p_{1}=p_{2}=\ldots=p_{m}>\max \left(p_{m+1}, \ldots, p_{n}\right)$ then $\dot{H}<0$ until $x_{i}=0$ for all $i \geq m$. Since there is no flux through the coordinate planes $x_{i}=0, i=1, \ldots, n$ then the equation (10) implies that the phase curve of (9) with a positive initial value remains forever in the area of $R_{+}^{n+1}$ bounded by the surface $H\left(x_{1}, \ldots, x_{n}, y\right)=$ $H\left(x_{1}(0), \ldots, x_{n}(0), y(0)\right)$, and the $n-m$ coordinate planes $x_{i}=0, i=m+1, \ldots, n$.

If $m=n$ (that is $p_{1}=p_{2}=\ldots=p_{n}$ ), then the equation $H\left(x_{1}, \ldots, x_{n}, y\right)=$ constant is the first integral of (9).

Define further $n(n-1) / 2$ Lyapunov functions 


$$
V_{i j}=\frac{x_{j}^{b_{i}}}{x_{i}^{b_{j}}}
$$

where $i<j$. For every $V_{i j}$ we have

$$
\dot{V}_{i j}=\left(a_{j} b_{i}-a_{i} b_{j}\right) V_{i j}=b_{i} b_{j}\left(p_{j}-p_{i}\right) V_{i j}=\Delta_{i j} V_{i j} .
$$

Consequently

$$
V_{i j}(t)=V_{i j}(0) \exp \left(\Delta_{i j} \cdot t\right) .
$$

Since $p_{1}=p_{2}=\ldots=p_{m}>\max \left(p_{m+1}, \ldots, p_{n}\right),(12)$ implies that if $i \leq m, j>m$, then $\lim _{t \rightarrow \infty} V_{i j}=0$. Consequently, since the solutions are bounded, this implies that $\lim _{t \rightarrow \infty} x_{j}(t)=0$.

Since $p_{1}=p_{2}=\ldots=p_{m}$, all $m(m-1) / 2$ equations $V_{i j}\left(x_{i}, x_{j}\right)=$ constant, where $i<j \leq m$, are the first integrals of (9). It is easy to show that $m-1$ independent first integrals, for instance $V_{1 j}\left(x_{1}, x_{j}\right), j=2, \ldots, n$, may be chosen among them.

This completes the proof.

The system with one prey and $n$ predators, and with the assumption that direct interspecies competition between the predators is negligible, may be described by the equations

$$
\begin{gathered}
\dot{x}=x\left(a-\sum_{i=1}^{n} b_{i} y_{i}\right), \\
\dot{y}_{1}=y_{1}\left(-c_{1}+d_{1} x\right), \ldots, \dot{y}_{n}=y_{n}\left(-c_{n}+d_{n} x\right) .
\end{gathered}
$$

The order of the subscripts for (13) is chosen so that $q_{1} \leq q_{2} \leq \ldots \leq q_{n}$, where $q_{i}=d_{i} / c_{i}$ is the survival capacity of $y_{i}$. Then the extension of Lemma 1 holds for (9) and (13).

Lemma 2 The systems (9) and (13) are equivalent under inversion of time $t$.

The proof is straightforward, and we omit it here.

The following extension of Theorem 2 to the system (13) is the direct consequence of Theorem 3 and Lemma 2.

THEOREM 4 The solutions of (13) with positive initial values are bounded, and, if $q_{n}=q_{n-1}=\ldots=q_{n-m+1}>\max \left(q_{1}, \ldots, q_{n-m}\right)$, then (13) has $m-1$ independent first integrals, and $\lim _{t \rightarrow \infty} y_{i}=0$ for all $i \leq n-m$.

If $q_{n}>\max \left(q_{1}, \ldots, q_{n-1}\right)$, then $\lim _{t \rightarrow \infty} y_{i}=0$ for all $i<n$.

The proof is analogous to that of Theorem 3 .

\section{Discussion}

The results of this paper demonstrate that, under the assumptions made, a biological species may be eliminated (effectively in finite time). A herbivorous species 
can be eliminated by predation if there is another herbivorous species with a higher survival capacity and which provides a predator with a permanent food base, even if there is no direct competition between these two herbivorous species. An unsuccessful predator will be eliminated in finite time if there is another predator with higher survival capacity. The results lead to the conclusion that predation, not competition only, is responsible for extinction of some species in the process of evolution.

It has been mentioned already that, as a consequence of the artificial introduction of alien species into New Zealand, there are some examples of the systems considered in this paper. Our results lead to the unfavourable conclusion that the New Zealand indigenous species are definitely driven to extinction, not only in consequence of unsuccessful competition, but even when competition is absent. For example, in the above-mentioned case (kiwi-rabbits-stoats system) the kiwi may be completely eliminated in finite time by predation alone.

The results in this paper suggest a new approach to a problem of a pest control. Usually biological pest control requires the introduction of a predator decreasing the pest population to an acceptable level. This approach provides only a short term result as after some time this predator-prey system has reached its coexisting equilibrium. According to our results, pest control would be much more effective if, simultaneously with the introduction of predators, a prey species is introduced to provide the predators with a long-term food base. It is not necessary for the introduced prey to compete with the pest for resources. It is even better if they do not compete, as the resources can be the subject of protection. But the introduced prey species must have a higher survival capacity than the pest has. Under these circumstances, the original pest species will be completely annihilated in finite time. Of course, the introduced alternative prey should be harmless or less harmful than the original one.

Though the analysis in this paper uses the simplest predator-prey model an interesting biological result have been derived. Consequently, it will be worthwhile to examine the robustness of this result in more detailed models.

\section{Acknowledgments}

We thank Dr R. Chan, University of Auckland and Dr M. Nelson, University of Leeds for useful discussions and their assistance with making this paper ready for publication.

\section{References}

1. G.J. Butler. Competitive predator-prey systems and coexistence. Population Biology, Lecture Notes in Biomath. Springer, Berlin, 52: 211-217, 1983.

2. K. Fujii. Complexity-stability relationship of two-prey-one-predator species system model: local and global stability. J. Theor. Biol., 69: 613-623, 1977. 
3. B.-S. Goh. Management and Analysis of Biological Populations. Elsevier Science, Amsterdam, 1980.

4. S.B. Hsu. Predator-mediated coexistence and extinction. Math. Biosci, 54: 231-248, 1981.

5. N. Krikorian. The Volterra model for three species predator-prey systems: boundedness and stability. J. Math. Biol., 7: 117-132, 1979.

6. Z. Lu. Global stability for a Lotka-Volterra system with a weakly diagonally dominant matrix. Appl. Math. Lett., 11(2):81-84, 1998.

7. Y. Takeuchi. Global Dynamical Properties of Lotka-Volterra Systems. World Scientific, Singapore, 1996.

8. Y. Takeuchi and N. Adachi. Oscillations in predator-prey Volterra models, in Population Biology, Lecture Notes in Biomath. Springer, Berlin, 52: 321-326, 1983.

9. R.R. Vance. Predation and resource partitioning in one predator-two prey model communities. Amer. Nat., 112: 797-813, 1978. 


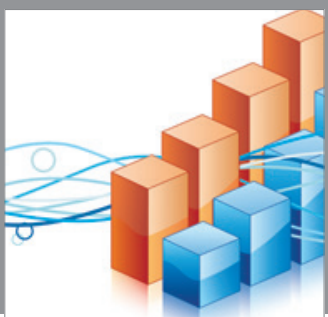

Advances in

Operations Research

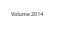

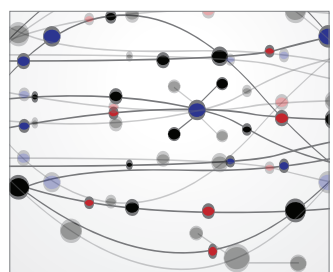

\section{The Scientific} World Journal
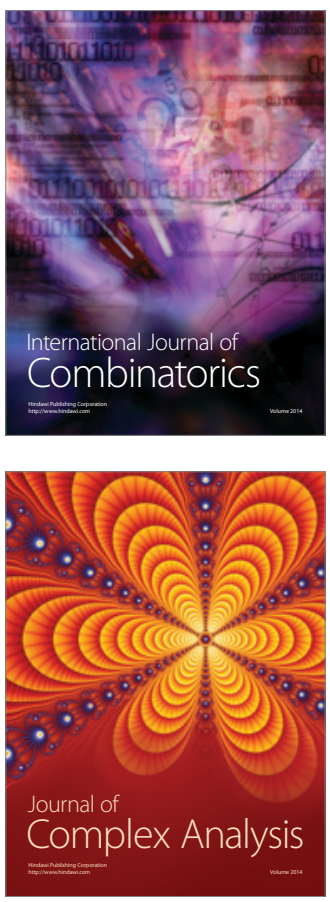

International Journal of

Mathematics and

Mathematical

Sciences
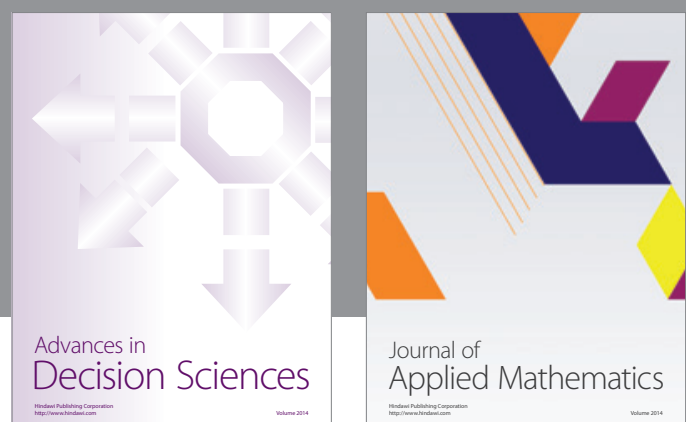

Journal of

Applied Mathematics
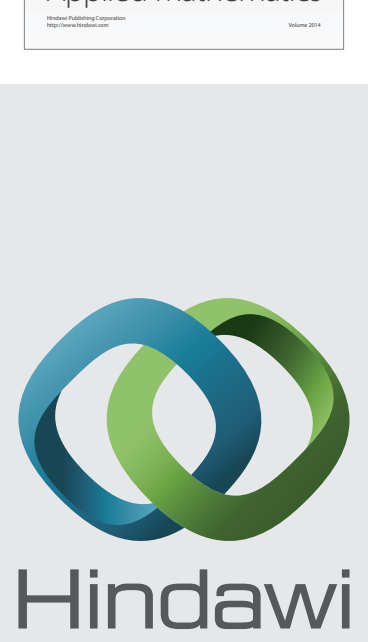

Submit your manuscripts at http://www.hindawi.com
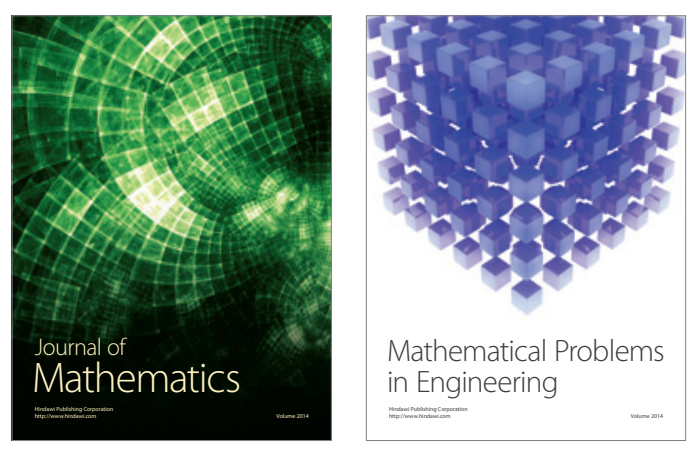

Mathematical Problems in Engineering
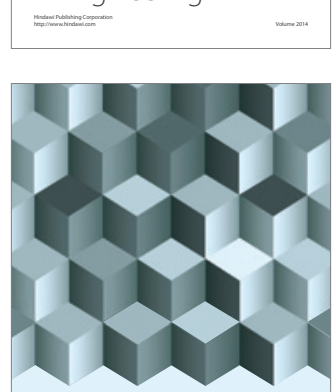

Journal of

Function Spaces
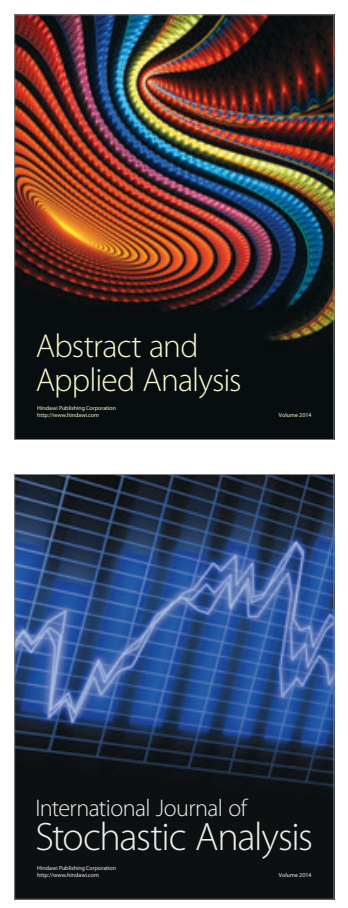

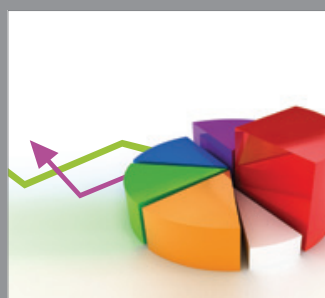

ournal of

Probability and Statistics

Promensencen
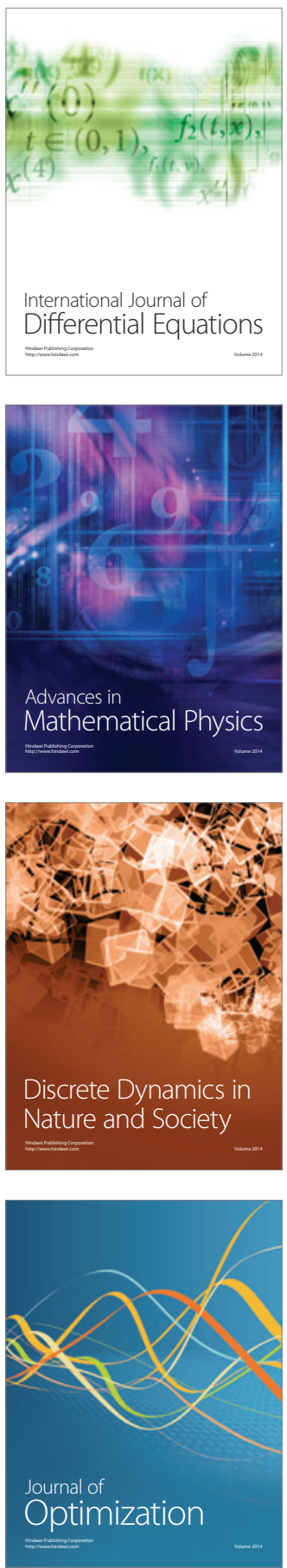